\title{
Alulah Taibel (1892-1984) a remarkable ornithologist, aviculturist and zoo-biologist
}

\author{
Spartaco Gippoliti
}

\begin{abstract}
Riassunto - Alulah Taibel (1892-1984): un rimarchevole ornitologo, avicoltore e biologo di zoo.

Alulah Taibel è stato uno zoologo italiano di origine austriacoyemenita. Terminata la sua carriera militare dopo la Prima Guerra Mondiale e laureatosi in Scienze Naturali a Bologna nel 1925, è stato direttore della Stazione di Pollicoltura di Rovigo e direttore scientifico del Giardino Zoologico di Torino. Mentre la sua interessante ma poco conosciuta esperienza bellica è stata oggetto di una recente monografia, la sua opera scientifica non è stata mai motivo di discussione dopo la sua scomparsa. Nel presente contributo la sua attività scientifica è sinteticamente illustrata e si presenta l'elenco completo delle sue pubblicazioni scientifiche.
\end{abstract}

Parole chiave: Cracidae, sistematica, consanguineità, Alessandro Ghigi.

\begin{abstract}
Alula Taibel has been an Italian zoologist of Austrian-Yemenite origin. After having served the Italian Army, he graduated in Natural Sciences at Bologna University in 1925. He was later director of the Poultry Station at Rovigo and scientific director of the Turin Zoological Garden. As to our knowledge no overview of his scientific work has never been published, a synthesis is here presented together with an almost complete list of his papers. Taibel has been one of the major experts on the Galliformes of the Cracidae family and therefore his papers on them are of particular relevance for ornithologists.
\end{abstract}

Key words: Cracidae, systematic, inbreeding, Alessandro Ghigi.

A recently published book, Alula Taibel, ardito $e$ naturalista (Bollini \& Bragatto, 2019) deals with the complicated personality of Alulah Taibel (or Alula M. Taibel and also sometimes Taibell), a unique figure among the Italian ornithological world. Alula Antonio Taibel (from his official birth certificate) born in Copparo (Ferrara) on 8 January 1892 from Gondisalvo Taibel and Giuditta Tagliaferri. For an understanding of

Società Italiana per la Storia della Fauna "Giuseppe Altobello", Viale Liegi 48, 00198 Roma, Italia.

E-mail: spartacolobus@hotmail.com

Received: 27 October 2019

Accepted for publication: 17 April 2020

Online publication: 20 May 2020 the etymology of his first name and the history of the Taibel family, readers are invited to see the cited book (Bollini \& Bragatto, 2019). In the present contribution, the choice has been done to use Alulah because this was clearly the wish of Taibel "to agree with Yemenite wording". Furthermore, he added a "M." in his name that has no official support in available documents, but should means "Mohamed" and signals his acceptance of Muslim religion, as reported by some testimonies. Instead of concentrating his interest towards Italian avifauna, Alulah was influenced by his mentor Alessandro Ghigi to pursue systematic studies of the world avifauna through avicultural activities and breeding experiments (Benazzi, 1984). He resumed his studies in Natural History at Bologna University in 1923, after being one of the more decorated Italian militaries during the Italian-Turkish war in Libya (1911) and the First World War. He graduated in Natural History in 1925. Despite this later achievement (he was 33 years old then) he was a particularly productive researcher still publishing well in his eighties. Furthermore, Taibel's skills included field expeditions and preparation and handling of both living and museum specimens, as evidenced by his Guatemala expedition in 1932 . This first-class naturalist also graduated in veterinary in 1932 and became later a leading figure of the zoo world in Italy (Gippoliti \& Violani, 2009). In 1924 he began working in the Experimental Poultry Station in Rovigo under Ghigi's direction. Here he also began to breed a number of exotic birds and mammals. In 1936-1937 he was consulting zoologist at the Giardino Zoologico in Rome and in 1938-1939 was in India with the animal trader Arduino Terni. In 1939 he could not access to a chair in genetics because he lacked the membership to the fascist party. In the same year, Taibel was sent to Ethiopia, then under Italian occupation, to capture the mythical Mountain nyala Tragelaphus buxtoni for the Zoological Garden in Rome. As he was overtaken there by the beginning of the Second Word War, he resumed his military duty as Colonel and after surviving a serious fighting with Ethiopians irregular, was captured by the English Army and made a prisoner of war in India.

As he returned to Italy in 1946, he resumed his work as director of the Rovigo Poultry Station. In 


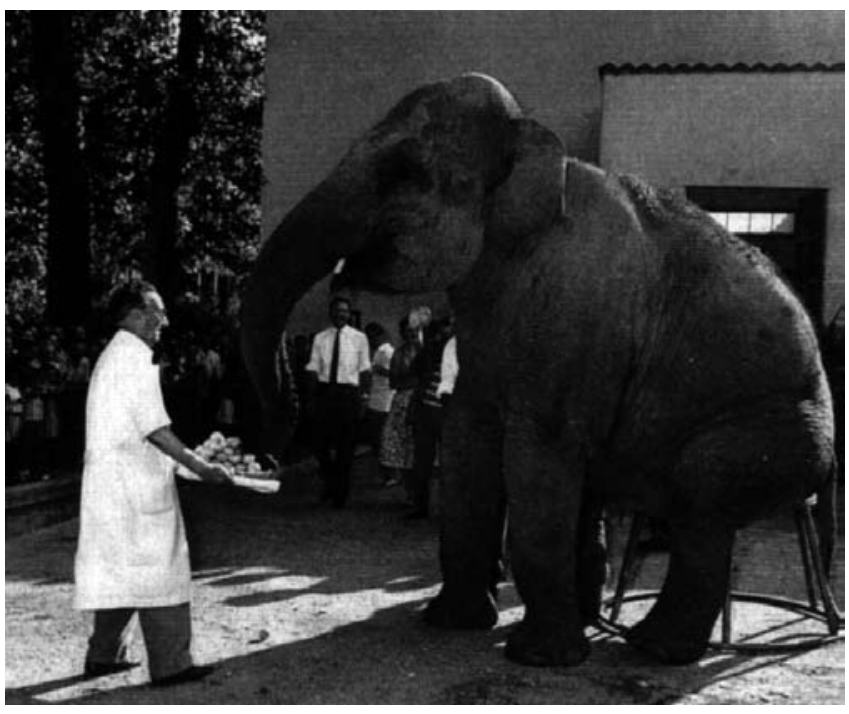

Fig. 1 - Alulah Taibel al Giardino Zoologico di Torino. / Alulah Taibel at the Turin Zoo. (Foto / Photo: Archivio Terni).

1953 he spent two months in Central Africa always associated with a capture expedition with the Molinar brothers, a famous animal trade agency based in Turin. From 1955 Taibel also took up the scientific direction of the zoos of Turin and Milan, both managed by the Molinar family at the time associated with Arduino Terni. He was certainly the right man in the right place (cf. Gippoliti \& Kitchener, 2007). Between 19551958 he produced a scientific bulletin of the two zoos 'Zoo - Bollettino dei Giardini Zoologici di Milano e Torino' that still remain a unique experiment in Italy. Here he published a wealth of papers mainly dealing with cracids breeding and systematics, his preferred subject of investigation. In other papers Taibel reviewed his hybridization experiments among members of the different genera Crax, Mitu, Pauxi and Penelope and on the basis of their inter-fecundity he proposed to treat all these cracids as members of a unique genus, Crax, with one superspecies and four species recognized following the Biological Species Concept of Mayr (Taibel, 1954). Similar results he obtained with other genera, and although he did not departed from Mayr's orthodoxy, he noted that some cracid 'species' seemed morphologically and geographically the result of hybridization between two other species. During such experiments he studied how inbreeding could lead to the development of lethal abnormalities, such as an Lshaped tibia (Taibel, 1971), well before this issue became a top concern in zoos and wildlife conservation (Ralls \& Ballou, 1983).

In Turin Zoo Taibel planned a series of aviaries in order to continue research on his preferred subject. Although some of his plans were never fully carried out, such as a research station off public view, he served as responsible of a 'Sperimental Section' until 1964 and he was the scientific planner of the highly appreciated Aquarium-Reptilarium realized by architect Venturel- li in 1960. Taibel planned a series of exhibit ordinate according a biogeographic and ecological criteria (Taibel, 1962), an exhibition style that would become more common in the following years. In this period an important primatological center was established in the Zoo in 1962, directed by the Anthropologist Brunetto Chiarelli of Turin University. Although birds were his first interest, he did not forget totally mammals. Among his studies on captive mammals it is worth to remember his papers on semi-free ranging Antilope cervicapra at Rovigo (Taibel, 1937) and a detailed morphological and anatomical description of capybara newborns (Taibel, 1950).

Although mainly a breeder and zoo-man, Taibel was a complete naturalist and researcher well aware of the importance of natural history collections stored in public museums. His 1932 Guatemala collections were deposited at the Zoological Museum of Bologna University and six new species of arachnids were described by Di Caporiacco. Bird and mammal specimens were later moved to the nearby Laboratorio di Caccia at Ozzano Emilia (now ISPRA Zoological Museum). His scientific perseverance is amply demonstrated by the publication of the mammalogical results of the Guatemala expedition in 1977, when he was 85 years old (Taibel, 1977)! A Taibel ornithological collection was also donated to the Museo civico di Zoologia in Rome and still awaits cataloguing. Among his last works, he also published a book for children in 1979, Leggende di Uccelli.

Taibel's papers had limited diffusion in Italy (unsurprisingly, considering the current emphasis on local ornithological studies) but - although written in Italian - some are still cited in international literature. This is the case with a paper dealing with the phyletic relationship of the Congo peafowl (Kimball et al., 1997) or with another paper dealing with some mysterious cracid taxa (Joseph et al., 1999).

Being Taibel a prolific writer and researcher, it is considered worthwhile to provide here a list of all scientific papers produced by him and known to us. Often these papers have been published on journals that are not easy to find even in Italy and hopefully the present effort may be useful to foreign scientists working on the same issues that Taibel studied with such dedication for more than half a century. The full collection of Taibel's papers is deposited in the library at the former Laboratorio di Caccia (now ISPRA Zoological Museum) at Ozzano Emilia (Bologna).

\section{Acknowledgements}

I wish to thank Giacomo Bollini that furnished me with the long-awaited input to remember Alulah Taibel to the international scientific community. Nicola Baccetti and Adriano De Faveri kindly supported our request of information about Taibel's materials at the ISPRA laboratory. Carlo Violani furnished a critical revision of the manuscript and Paola Di Luzio helped me with researches at the Library of the Museo civico di Zoologia in Rome. 


\section{LIST OF TAIBEL'S PUBLICATIONS}

Taibel A. M., 1926 - La Stazione sperimentale di Pollicoltura di Rovigo. L'Italia Agricola, novembre 1926: 299-332.

Taibel A. M., 1926 - Le migliori razze di galline. L'Italia Agricola, novembre 1926: 3-16.

Giacomini E. \& Taibel A. M., 1927 - Sulle modificazioni del piumaggio causate dalla tiroide. Bollettino della Società Italiana di Biologia Sperimentale, 2 (5): 4-27.

Giacomini E. \& Taibel A. M., 1927 - Gli effetti della tiroide sulla muta e sulla deposizione delle galline. Bollettino della Società Italiana di Biologia Sperimentale, 2 (5): 28-36.

Giacomini E. \& Taibel A., 1927 - Gli effetti della tiroide sulla muta e sulla deposizione delle galline. Rendiconti delle Sessioni della Reale Accademia delle Scienze dell'Istituto di Bologna, marzo 1927: 460-463.

Giacomini E. \& Taibel A. M., 1927 - Sulle modificazioni del piumaggio causate dalla tiroide e particolarmente su quelle presunte sessuali nei polli ipertiroidizzati. Rendiconti delle Sessioni della Reale Accademia delle Scienze dell'Istituto di Bologna, marzo 1927: 465-468.

Ghigi A. \& Taibel A. M., 1927 - Récherches sur l'hérédité de la couleur et d'autres caractères chez les canards. Report of Proceedings of III International Poultry Congress, Ottawa.

Ghigi A. \& Taibel A. M., 1927 - Resultats obtenus par le croisement des races Indian Game et Italienne Blanche. Report of Proceedings of III International Poultry Congress, Ottawa.

Taibel A. M., 1927 - Augmentation de poids des poussins proportionellement à la qualité de Hydrates de carbone dans leur ration complete par de la farine de chrysalide du ver à soi. Report of Proceedings of III International Poultry Congress, Ottawa.

Taibel A. M., 1928 - Influenza della tiroide sul piumaggio della quaglia. Rivista di biologia, 10 (3-4): 4-18.

Taibel A. M., 1928 - Risveglio artificiale di istinti tipicamente femminili nei maschi di taluni uccelli. Atti della Società dei Naturalisti e Matematici di Modena, (6) 7: 2-10.

Taibel A. M., 1928 - L'eredità mendeliana dell'albinismo in Phasianus colchicus. Rivista di Biologia, 10 (5-6): 4-9.

Taibel A. M., 1929 - Anomalie nel becco degli uccelli. Natura (Milano) 20: $4-8$.

Taibel A. M., 1929 - Recensione critica del lavoro di Masauji Hachisuka: Variationns among birds. The Ornit. Soc. of Japan, 1928. Rivista di biologia, 11 (3-4): 2-3.

Taibel A. M., 1929 - Osservazioni di genetica su Fringillidi. Nota I. Natura (Milano), 20: 66-68.

Taibel A. M., 1929 - Osservazioni di genetica su Fringillidi. Nota II. Natura (Milano), 20: 140-151.

Taibel A. M., 1930 - Fattori multipli in due tipi antagonistici di cresta nei polli. Bollettino di Zoologia, 1 (1): 21-25.

Taibel A. M., 1930 - Arrenoidia di una femmina Gennaeus lineatus Vigors, in seguito ad involuzione ovarica. Rivista di biologia, 12 (1-2): 3-15.

Taibel A. M., 1930 - Ricerche sulla eredità di alcuni fattori nelle anatre domestiche. Pubblicazione della Stazione Sperimentale di Pollicoltura di Rovigo, 5: 5-36.

Taibel A. M., 1930 - Modificazioni insorgenti nelle penne del gallo in seguito alla emasculazione. Pubblicazione della Stazione Sperimentale di Pollicoltura di Rovigo, 6: 5-15.

Taibel A. M., 1930 - Come viene risolto il problema della pulizia del nido in talune specie di uccelli a prole inetta. Natura, 21: 1-7.

Taibel A. M., 1930 - Ibridi artificiali interspecifici nel genere Streptopelia. Atti della Società Italiana di Scienze Naturali e del Museo Civico di Storia Naturale in Milano, 69: 1-24.

Taibel A. M., 1930 - Selection of egg-laying stock from the crossing of pure leghorn stock with local Italian breeds. IV International Poultry Congress, London: 72-77.

Taibel A. M., 1930 - Sul cavallo di Rodi. Bollettino di Zoologia, 1 (4): 128-130.

Taibel A. M., 1930 - La gonomonarrenia negli ibridi Streptopelia turtur (L.) x Streptopelia decaocto (Friv.). Rivista di biologia, 12: 3-15.

Taibel A. M., 1930 - Un ibrido di tipo eccezionale nell'incrocio di due varietà di polli di razza italiana. Bollettino di Zoologia, 1 (6): 285 289.
Taibel A. M., 1931 - Eredità legata al sesso in varietà e specie del genere Streptopelia Bp. Archivio Zoologico Italiano, 16: 384-392.

Taibel A. M., 1931 - Eredità di caratteri legati al sesso in alcune varietà e specie di tortore del genere Streptopelia Bp. Archivio Zoologico Italiano, 17: 103-121.

Taibel A. M., 1931 - IV Congresso Mondiale di Avicultura. Rivista di biologia, 13 (1-3): 1-6.

Taibel A. M., 1931 - Arrenoidia parziale di una gallina domestica senza scomparsa della attività ovarica. Rivista di biologia, 13 (1-3): $3-15$.

Taibel A. M., 1931 - Alcune osservazioni intorno ai maiali dell'Isola di Piscopi, Rivista di biologia, 8: 3-13.

Taibel A. M., 1931 - L'allevamento delle Volturine. Rivista di Avicoltura, 1 (6): 4-14.

Taibel A. M., 1932 - Risultati di incrocio Columba domestica x Columba guinea. Zeitschrift für induktive Abstammungs- und Vererbungslehre, 61 (2): 301-312.

Taibel A. M., 1932 - L'istinto delle cure parentali di ibridi Phasianus x Gallus completamente sterili. Rivista di biologia, 14 (1-2): 3-11.

Taibel A. M., 1932 - A proposito della cattura di un beccacino col ciuffo. Rivista Italiana di Ornitologia , (2) 3: 2-3.

Taibel A. M., 1933 - L'istinto migratorio carattere mendeliano recessivo in Streptopelia. Natura, 24: 51-53.

Taibel A. M., 1933 - Alla ricerca del Tacchino ocellato. Rivista di Avicoltura, 3: 3-14.

Taibel A. M., 1933 - Interpretazione del fenomeno della comparsa di eccezioni alla regola della eredità legata al sesso in alcune specie di uccelli. Archivio Zoologico Italiano, 19: 467-479.

Taibel A. M., 1933 - Valore genetico del colore grigio-azzurro del piumaggio del tacchino domestico. Rivista di Avicoltura, 3 (3): 1-5.

Taibel A. M., 1933 - Osservazioni sulla fecondità delle galline comuni romagnole. Rivista di Avicoltura, 3 (10): 1-4.

Taibel A. M., 1934 - Esperimento di bursafabriciectomia in Gallus domesticus. Atti V Congresso Mondiale Pollicoltura, Roma: 5-19.

Taibel A. M., 1934 - Contributo alla sistematica della famiglia Columbidae. Rivista di biologia, 16 (1): 3-16.

Taibel A. M., 1934 - Il tacchino ocellato di Guatemala. Rivista Italiana di Ornitologia, (2) 4: 103-112.

Taibel A. M., 1934 - Quadro sintomatologico somato-psichico proprio della gravidanza e del puerperio in femmine non fecondate. Contributo all'ipotesi di un contributo del sistema nervoso nella determinazione del parto. Rivista di biologia, 17 (2): 3-11.

Taibel A. M., 1934 - Le razze locali alla XI Mostra animali da cortile di Padova. Rivista di Avicoltura, 4: 3-9

Taibel A. M., 1935 - Contributo alla casistica dello studio delle neoplasie negli uccelli. Rivista di Avicoltura, 5: 1-4.

Taibel A. M., 1935 - Osservazione intorno ai gruppi di galline per il concorso deposizione uova. Italia Agricola, 72 (3): 3-16.

Taibel A. M., 1935 - Appunti ornitologici (Zoologia generale). Rivista Italiana di Ornitologia, (2) 5: 1-8.

Taibel A. M., 1935 - Risultati d'incrocio Columba picazuro x Columba domestica. Bollettino di Zoologia, 6 (1-2): 171-186.

Taibel A. M., 1935 - Appunti ornitologici (Genetica). Rivista Italiana di Ornitologia, II-5 (1): 203-212.

Taibel A. M., 1935 - Esperimento di bursafabriciectomia in Gallus domesticus. Rivista di biologia, 18 (3): 439-443.

Taibel A. M., 1935 - Duplicità degli uccelli. Rivista di biologia, 19 (1): 3-16.

Taibel A. M., 1935 - Lo sprone nelle galline. Rivista di Avicoltura, 5 (4): 205-207.

Taibel A. M., 1936 - Sprone e sesso. Rivista di biologia, 21 (1): 3-23.

Taibel A. M., 1936 - Recensione critica del lavoro faraone e tacchini di Ghigi A. Rivista di biologia, 20 (2): 1-3.

Taibel A. M. \& Levi G., 1936 - Un caso di persistenza dell'ovidutto destro in Phasianus colchicus. Bollettino di Zoologia, 7 (4): 137-143.

Taibel A. M., 1937 - Ibridi Trichoglossus ornatus x T. novae-hollandiae. Rivista Italiana di Ornitologia, II-7 (1): 1-12.

Taibel A. M., 1937 - Recensione critica del lavoro Monografia dei fagiani di Ghigi A. Rivista di Biologia, 23 (3): 1-3.

Taibel A. M., 1937 - L'antilope cervicapra: osservazioni sul gruppo in allevamento presso la Stazione sperimentale di Avicoltura di Rovigo. Rassegna Faunistica, 4: 3-19. 
Taibel A. M., 1937 - Anomalie nel colorito del mantello in alcuni Mammiferi del Giardino Zoologico di Roma. Bollettino di Zoologia, 8: 211-217.

Taibel A. M., 1937 - Sulla ricostruzione della razza Valdarno e sulla sua selezione. Rivista di Avicoltura, 10: 3-7.

Taibel A. M., 1938 - Effetto della bursectomia sul timo in Gallus domesticus. Rivista di biologia, 24: 3-11.

Taibel A. M., 1938 - Terre cotte zoomorfiche precolombiane. Natura, 29: $1-8$.

Taibel A. M., 1938 - Appunti ornitologici (Teratologia). Rivista Italiana di Ornitologia, II-8 (2): 46-65.

Taibel A. M., 1938 - Ulteriori osservazioni sulla selezione di galline comuni Romagnole. Rivista di Avicoltura, 6: 3-11.

Taibel A. M., 1938 - Sur l'elevage en captivite du Tinamus major robustus Sclater and Salvin. In: Proceedings of the IX International Ornithological Congress. J. Delacour (ed.). Le cerf, Rouen: 373379.

Taibel A. M., 1938 - Le cure parentali in Crax globicera Lin. Rivista di Avicoltura, 12: 3-7.

Taibel A. M., 1938 - Ricerche di ibridismo nel genere Anas L. Atti III Riunione Società Italiana di Genetica e di Eugenetica, Bologna, sett. 1938: 3-5.

Taibel A. M., 1939 - Ibridi artificiali interspecifici nel genere Streptopelia Bp. Atti della Società Italiana di Scienze Naturali e del Museo Civico di Storia Naturale in Milano, 78: 3-31.

Taibel A. M., 1939 - Notizie sull'allevamento in cattività di Tinamus major robustus Sclater. Rivista Italiana di Ornitologia, 9: $1-31$.

Taibel A. M., 1939 - Lucknow: mercato di fagiani del Nepal. Rivista di Avicoltura, 5: 3-5.

Taibel A. M., 1939 - Un caso di poliuroptilia (timoniere soprannumerarie) in Crax globicera globicera-. Bollettino di Zoologia, 10 (3-4): 191-195.

Taibel A. M., 1939 - Nuova mutazione somatic in Pavo cristatus L. Bollettino di Zoologia, 10 (3-4): 181- 189.

Taibel A. M., 1940 - Osservazioni sulla riproduzione in cattività di Crax globicera globicera Linneus. Rivista Italiana di Ornitologia, 10: 93-126.

Taibel A. M., 1941 - Il timo nei polli bursectomizzati. Rivista di biologia, 31: 3-18.

Taibel A. M., 1945 - On experiments in albinism with chital Axis axis. Journal of the Bombay Natural History Society, 45: 417-419.

Taibel A. M., 1947 - Esperimento di bursa-timectomia in Gallus gallus domesticus L. Rivista di Biologia, 39: 1-26.

Taibel A. M., 1947 - Sterilità degli ibridi Columba rufina pallidicrissa x C. livia gaddi. Rivista Italiana di Ornitologia, 17: 147-150.

Taibel A. M., 1947 - Prime osservazioni sulla trasmissibilità della poliuroptilia in talune specie del genere Crax L. Bollettino di Zoologia, 16: 1-7.

Taibel A. M., 1948 - Ulteriori osservazioni sull'esplicazione delle cure parentali negli uccelli del genere Crax L. Rivista Italiana di Ornitologia, 18: 3-7.

Taibel A. M., 1948 - Di una anomalia anatomica negli arti di un Capibara, Bollettino di Zoologia, 15: 111-114.

Taibel A. M., 1948 - Osservazioni biologiche sopra tre giovani esemplari di Niala di Monte Tragelaphus buxtoni Lidd.) allevati per la prima volta in cattività. Bollettino di Zoologia, 15: 123-144.

Taibel A. M., 1949 - Una nuova razza di faraona domestica (Numidia meleagris L.) apparsa per mutazione. Scientia Genetica, 3: 172182.

Taibel A. M., 1949 - Sensibilità emotiva in Gallus gallus domesticus L. nella espressione mimica e vocale. Rivista Italiana di Ornitologia, 19: 53-68.

Taibel A. M., 1949 - Influenza della asportazione della cresta e dei bargigli sul peso dei testicoli e sulla spermatogenesi. Annali della Sperimentazione Agraria, n.s., 3: 327-342.

Taibel A. M., 1949 - Recensione critica del lavoro "problemi biologici della sessualità" di M. Benazzi. Bollettino della Società Italiana di Biologia Sperimentale, 33: 1-11.

Taibel A. M., 1949 - Ibridi artificiali interspecifici nel genere Streptopelia Bp. Nota III. Atti della Società Italiana di Scienze Naturali e del Museo Civico di Storia Naturale in Milano, 88: 171- 190.
Taibel A. M., 1949 - Inusitata ubicazione di un nido di Turdus merula (L.). Rivista Italiana di Ornitologia, 19: 167- 171.

Taibel A. M., 1949 - Nuovi risultati d'incrocio diretto e reciproco fra Columba livia domestica e Columba guinea. Archivio Zoologico Italiano, 34: 431-476.

Taibel A. M., 1949 - Su alcuni ibridi intergenerici Mitu x Crax. Bollettino di Zoologia, 16: 71-78.

Taibel A. M., 1949 - Un caso di poliuroptilia in Mitu mitu L. Bollettino di Zoologia, 16: 95-96.

Taibel A. M., 1950 - Osservazioni su alcuni neonati di capibara (Hydrochoerus hydrochoeris). Cesalpinia, gennaio: 29-47.

Taibel A. M., 1950 - Esperimenti ibridologici tra specie del genera Crax L. Cesalpinia, maggio: 49-93.

Taibel A. M., 1950 - Genesi della specie Crax viridirostris Sclater, alla luce della sperimentazione ibrido logica. Bollettino di Zoologia, 17 (4): 543-547.

Taibel A. M., 1951 - Un caso di assenza congenita delle remiganti primarie e secondarie in una gallina della razza New Hampshire. Annali della Sperimentazione Agraria, 5 (5): 1095-1099.

Taibel A. M., 1951 - Prole inetta e prole precoce negli uccelli. Etologia, sistematica, filogenesi. Cesalpinia, 1-88.

Taibel A. M., 1951 - Action dévirilisant des oestrogènes synthétiques. IX Congrès Mondial d'Aviculture, Paris.

Taibel A. M., 1951 - La tortora dal collare orientale - Streptopelia d. decaocto (Friv.) - avvistata anche nel Polesine - accertata nidificazione a Rovigo. Rivista Italiana di Ornitologia, 21: 137-150.

Taibel A. M., 1951 - Streptopelia risoria, S. decaocto, S. douraca, S. roseogrisea. Chiarificazioni sulla nomenclatura e sulla sistematica. Bollettino di Zoologia, 18: 4-6.

Taibel A. M. \& Ghiara F., 1951 - Insorgenza di alcuni caratteri ginoidi in un gallo di razza livornese bianca sottoposto a trattamento di estrogeno sintetico (dietilstilbestrolo). Annali della Sperimentazione Agraria, 5 (4): 895-900.

Taibel A. M., 1953 - Nuova varietà di colore del piumaggio dell'anatra muschiata Cairina moschata domestica L. ottenuta per mutazione. Annali della Sperimentazione Agraria, 7 (6): 1-5.

Taibel A. M., 1953 - Osservazioni sulla riproduzione e allevamento in cattività di Penelope superciliaris superciliaris Temminck e Ortalis garrula garrula (Humboldt). Rivista Italiana di Ornitologia, 23: $85-122$.

Taibel A. M., 1953 - Comportamento genetico del nuovo carattere 'bar' nell'anatra muschiata (Cairina moschata domestica L.). Annali della Sperimentazione Agraria, 7 (7): 1-18.

Taibel A. M., 1954 - Il piumaggio 'grigio-ardesia-azzurrastro' dell'anatra muschiata (Cairina moschata domestica L.) e suo valore genetico. Creazione della varietà 'grigio-perla' per mutazione. Annali della Sperimentazione Agraria, 8 (1): 1-7.

Taibel A. M., 1954 - Osservazioni sulla esplicazione delle cure parentali negli uccelli dei generi Penelope e Ortalis. Natura, 45: 1-14.

Taibel A. M., 1954 - A group of plumage factors in the Muscovy duck (Cairina moschata domestica L.). X World's Poultry Congress, Edinburgh, 2.

Taibel A. M., 1954 - Sistematica e Ibridologia. Generi, Specie, Sottospecie, Razze biologiche nella Sottofamiglia Cracinae. Bollettino di Zoologia, 21 (2): 261-272.

Taibel A. M., 1954 - Notizie sulla riproduzione in cattività del Colombo dal collare bianco (Columba albitorques Rüppell). Rivista Italiana di Ornitologia, 24: 195-203.

Taibel A. M., 1955 - Su taluni ibridi o rari o nuovi alla scienza ibridologica. Rivista Italiana di Ornitologia, 25: 1-28.

Taibel A. M., 1955 - Gli uccelli del Guatemala con speciale riguardo alla regione del Petèn raccolti dal maggio al settembre 1932. Atti della Società Italiana di Scienze Naturali e del Museo Civico di Storia Naturale in Milano, 94: 15-84.

Taibel A. M., 1955 - Compiti e finalità di uno Zoo moderno. Zoo Bollettino dei Giardini Zoologici di Milano e Torino, 1: 15-20.

Taibel A. M., 1955 - Sistematica del gruppo Cracinae - Nota prima. Considerazioni intorno alla presunta specie Crax pinima Pelz. Zoo Bollettino dei Giardini Zoologici di Milano e Torino, 1: 21-32.

Taibel A. M., 1955 - La pecora di Soay - osservazioni sul gruppo in allevamento alla Stazione Sperimentale di Avicultura di Rovigo. Zoo Bollettino dei Giardini Zoologici di Milano e Torino, 1: 41-66. 
Taibel A. M., 1955 - Torino ha avuto il primo giardino zoologico italiano. Zoo Bollettino dei Giardini Zoologici di Milano e Torino, 1: 98-108.

Taibel A. M., 1955 - Perché vengono emanate leggi protettive sugli uccelli se poi non debbono venire osservate? Zoo Bollettino dei Giardini Zoologici di Milano e Torino, 1: 125.

Taibel A. M., 1956 - Osservazioni sulla 'muta' del pinguino reale (Aptenodytes patagonica) allo Zoo di Torino. Zoo Bollettino dei Giardini Zoologici di Milano e Torino, 2: 79-80.

Taibel A. M., 1956 - Risultati di incroci e reincroci fra Columbia livia domestica e C. albitorques. Zoo Bollettino dei Giardini Zoologici di Milano e Torino, 2 (3), 55-74; 99.

Taibel A. M., 1956 - La morte di una femmina di Crace globuloso (Crax globicera) all'età di 24 anni e 4 mesi. Zoo Bollettino dei Giardini Zoologici di Milano e Torino, 2: 86-89.

Taibel A. M., 1956 - Recensione critica a L'amico degli animali racconta... di Angelo Lombardi. Zoo Bollettino dei Giardini Zoologici di Milano e Torino, 2 (4): 1-6.

Taibel A. M., 1956 - Nuove mutazioni, causa di nuove varietà, nel corredo cromosomico dell'anatra muschiata (Cairina moschata domestica L.). Annali della Sperimentazione Agraria, 10 (6): 1-12.

Taibel A. M., 1957 - Osservazioni sulla riproduzione e allevamento in cattività di Penelope superciliaris superciliaris Temminck e Ortalis garrula garrula (Humboldt). Zoo Bollettino dei Giardini Zoologici di Milano e Torino, 3: 16-41.

Taibel A. M., 1957 - Sistematica del gruppo Cracinae. Nota seconda. Considerazioni critiche all'ordinamento sistematico-tassonomico di J.L. Peters. Zoo Bollettino dei Giardini Zoologici di Milano e Torino, 3: 65-78.

Taibel A. M., 1957 - Descrizione dell'ibrido maschile Crax fasciolata Spix x Crax alberti Fraser. Zoo Bollettino dei Giardini Zoologici di Milano e Torino, 3: 94-125.

Taibel A. M., 1957 - Alcuni dati ponderali su una cucciolata di Coati (Nasua nasua) nati allo Zoo di Milano e due cucciolate di Procioni (Procyon lotor) nati allo Zoo di Torino. Zoo Bollettino dei Giardini Zoologici di Milano e Torino, 3: 146-147.

Taibel A. M., 1957 - Analisi di un primo gruppo di fattori del corredo cromosomico dell'anatra muschiata (Cairina moschata domestica L.). Atti III Riunione AGI (Associazione Genetica Italiana): 3-7.

Taibel A. M., 1958 - Protezione della natura e Giardini Zoologici. Zoo Bollettino dei Giardini Zoologici di Milano e Torino, 4: 27-33.

Taibel A. M., 1958 - Presenza di un carattere "concordo sessuale" interessante il colore del mantello nel Coati (Nasua nasua). Zoo Bollettino dei Giardini Zoologici di Milano e Torino, 4: 34-42.

Taibel A. M., 1958 - Osservazioni su un allevamento in cattività di Oca lombardella (Anser albifrons). Zoo Bollettino dei Giardini Zoologici di Milano e Torino, 4: 1-7.

Taibel A. M., 1958 - Esperimenti ibridologici tra specie del genere Crax L. Memoria seconda. Ibridi Crax globicera x C. alberti F2 ed ibridi di reincrocio. Zoo. Bollettino dei Giardini Zoologici di Milano e Torino, 4: 127- 177.

Taibel A. M., 1958 - Un nuovo fattore di ripartizione causa della "pezzatura bianca al capo e al terzo superiore del collo" nel corredo cromosomico dell'anatra muschiata (Cairina moschata domestica L.). Annali della Sperimentazione Agraria, 10 (6): 537-553.

Taibel A. M., 1959 - Considerazioni sistematico-genetiche sulle famiglie Equidae e Ursidae. Bollettino di Zoologia, 26 (2): 483-504.

Taibel A. M., 1961 - Due nuove varietà di colore nel piumaggio dell'Anatra muschiata (Cairina moschata domestica L.) sorte per mutazione. Bollettino di Zoologia, 28 (2): 561-568.

Taibel A. M., 1961 - Analogie fisio-etologiche nel settore riproduttivo tra Afropavo Chapin e Penelope Merrem. Natura, 5, 57-64.

Taibel A. M., 1961 - Esperimento ibridologico tra specie e generi distinti Mitu e Crax. Nota prima: ibridi di prima generazione. Archivio Zoologico Italiano, 46: 181-226.

Taibel A. M., 1961 - Esperimento ibridologico tra specie e generi distinti Mitu e Crax. Nota seconda: Ibridi di seconda generazione e ibridi di re incrocio. Archivio Zoologico Italiano, 46: 291-324.

Mainardi D. \& Taibel A. M., 1962 - Studio immunogenetico sulle parentele filogenetiche nell'ordine dei Galliformi. Istituto Lombardo Accademia di Scienze e Lettere (Rendiconti di Scienze) B, 95: 131140.
Mainardi D. \& Taibel A. M., 1962 - Filogenesi dei Galliformi. Istituto Lombardo Accademia di Scienze e Lettere (Rendiconti di Scienze) $B, 96: 118-130$.

Taibel A. M., 1962 - Sistematica del gruppo "Cracinae". Bollettino di Zoologia, 29: 1, 35-47.

Taibel A. M., 1962 - Ricordo di Augusto Molinar. Giardino Zoologico (Roma), 4 (1): 30-31.

Taibel A. M., 1962 - L'Acquario-Rettilario dello Zoo di Torino. Natura e Montagna, (3): 133-138.

Taibel A. M., 1962 - Lo Zoo di Napoli. Natura e Montagna, (4): 182185.

Taibel A. M., 1962 - Considerazioni critiche su un presunto ibrido tra il Tacchino (Meleagris gallopavo) e la Faraona (Numida meleagris). Natura, 53: 57-68

Taibel A. M., 1962 - Ulteriori osservazioni sulla trasmissibilità della "poliuroptilia" in talune specie dei generi Crax e Mitu. Atti della Società Italiana di Scienze Naturali e del Museo Civico di Storia Naturale in Milano, 101: 167-180.

Taibel A. M., 1963 - Anche negli accurati lavori morfo-isto-citologici riguardanti gli Uccelli è necessario non venga trascurata la esattezza della terminologia sistematica. Rivista Italiana di Ornitologia, 33: 40- 42.

Taibel A. M., Mainardi D. \& Grilletto R., 1963 - Riconoscimento, mediante analisi immunogenetica, delle specie parentali di un presunto ibrido Ara chloroptera x Ara macao, e sua descrizione. Nuova Veterinaria, 39: 381-385.

Taibel A. M., 1964 - Ibridi Crax globicera $\times$ Crax faciolata $\mathrm{F}_{1}$ e considerazioni critiche intorno alle forme Crax hecki Reichenow Crax chapmani Nelson, Crax incommoda Sclater e Crax gryai OgilvieGrant. Archivio Zoologico Italiano, 49: 1-25.

Taibel A. M., 1964 - Esperimenti ibridologici tra specie del genere $P e$ nelope Merr. Nota seconda. Notizie sulla fertilità degli ibridi F1 $P$. purpurascens $\mathrm{x} P$. superciliaris; $\mathrm{P}$. purpurascens $\mathrm{x} P$. pileata; $P$. pileata x P. superciliaris. Rivista Italiana di Ornitologia, 34: 199212.

Taibel A. M., 1965 - Sistematica della famiglia "Cracidae". Archivio Zoologico Italiano, 50: 163-231.

Taibel A. M., 1966 - Una nuova varietà di colore - avorio - nel piumaggio della Tortora dal collare domestica (Streptopelia risoria L.) e suo comportamento genetico. Atti della Società Italiana di Scienze Naturali e del Museo Civico di Storia Naturale in Milano, 105 (2), 158-174.

Taibel A. M., 1966 - Descrizione di un ibrido femminile 'fertile' Crax alberti $\times$ Crax nigra F. Galliformes) ottenuto alla "Ménagerie du Jardin des Plantes" di Parigi. Bollettino di Zoologia, 33: 160 .

Taibel A. M., 1967 - Sistematica della famiglia Cracidae. Nota terza. Nuove considerazioni intorno al genere Penelope Merr. Atti della Società Italiana di Scienze Naturali e del Museo Civico di Storia Naturale in Milano, 106: 115-145.

Taibel A. M., 1967 - Problemi di sistematica biologica nell'ambito della famiglia Cracidae (Galliformes). Accademia Nazionale dei Lincei, Rendiconti. Classe di Scienze Morali, Storiche e Filologiche, (VII) 12: 948-954.

Taibel A. M. \& Calderisi A., 1967 - Ricerche sulla pigmentazione delle gonadi in uccelli appartenenti alla famiglia Cracidae (Galliformes). Rivista Italiana di Ornitologia, 37: 241-250.

Taibel A. M. \& Grilletto R., 1967 - Atavistic polydactyla in the forelimb of a lama (Lama glama) (Artiodactyla, Tylopoda). Der Zoologischer Garten NF, 33: 174-181.

Taibel A. M., 1968 - Riproduzione ed allevamento del Colombo imperiale verde delle Isole Nicobar (Ducula aenea nicobarica) realizzato per la prima volta in Italia. Rivista Italiana di Ornitologia, 38: $150-168$.

Taibel A. M., 1968 - Considerazioni critiche su un "presunto" ibrido Coturnix coturnix japonica maschio e Gallus gallus femmina. Atti della Società Italiana di Scienze Naturali e del Museo Civico di Storia Naturale in Milano, 107: 175-180.

Taibel A. M., 1969 - Osservazioni sulla riproduzione e allevamento di Pipile jacutinga (Spix) (Cracidae - Galliformes) realizzata per la prima volta con esemplari in cattività. Annali Museo Civico di Storia Naturale Giacomo Doria, 76-77: 33-52. 
Taibel A. M., 1969 - Riproduzione e allevamento in cattività di Mitu mitu (Linneo) (Galliformes, Cracidae) ottenuti per la prima volta anche in ltalia. Ricerche di Zoologia applicata alla caccia, 45: $1-26$.

Taibel A. M., 1969 - Trasferimento del «fattore di diluizione» dalla Tortora dal collare (Streptopelia risoria) - varietà «avorio» - alla Tortora luttuosa (Streptopelia decipiens) con creazione di una nuova varietà di colore. Natura, 60 (1): 32-40.

Taibel A. M., 1969 - Precisazioni anatomo-fisio-etologiche su Nothocrax urumutum - Galliformes-Cracidae in seguito ad osservazioni su uccelli tenuti in cattività. Rivista Italiana di Ornitologia, 39: 3848.

Taibel A. M., 1971 - Tibie piegate ad "angolo", mutazione condizionata da un fattore "letale" in Crax e in Mitu (Galliformes - Cracidae). Natura, 62: 197-209.

Taibel A. M., 1972 - Esperimenti ibridologici tra specie del genere Penelope Merr. Nota terza. Ibrido P. pileata x P. argyrotis e suoi derivati dall'accoppiamento con $P$. superciliaris. Rivista Italiana di Ornitologia, 42: 277-300.

Taibel A. M., 1972 - Descrizione di un ibrido femminile "fertile" Crax alberti X Crax nigra F1 ottenuto alla "Ménagerie du Jardin des Plantes" di Parigi (Galliformes). Natura, 63: 127-150.

Taibel A. M., 1972 - Tortore ornamentali e da voliera. Edagricole, Bologna.

Taibel A. M. \& Grilletto R., 1972 - Esperimento ibridologico nel genere Cygnus Bechstein. Ibridi F1 Cygnus melanocoriphus (Molina) x C. olor (Gmelin). Atti Accademia Nazionale dei Lincei, 53 (5): 471-476.

Taibel A. M., 1973 - Scuotimento e tremolio del capo, costume comune a molti Cracidae (Galliformes) interpretato come conseguenza di determinati stimoli emotivi. Natura, 64: 139-146.

Taibel A. M., 1973 - Esperimenti ibridologici tra specie del genere $P e$ nelope (Galliformes, Cracidae): Nota quarta. Controllata fertilità in entrambi i sessi degli ibridi trispecifici (Penelope pileata $\mathrm{x} P$. argyrotis) x Penelope superciliaris. Rivista Italiana di Ornitologia, 43: $650-658$

Taibel A. M., 1973 - Notizie biologiche sulla famiglia Cracidae (Galliformes). Nota seconda: il pulcino. Neonato e stadi immediatamente successivi. Supplemento alle Ricerche di Biologia della Selvaggina, 5 (5): 71-173.

Taibel A. M., 1973 - Considerazioni critiche sulle recenti osservazioni di C. Vaurie sull'ordinamento sistematico e alla nomenclatura seguite da J.L. Peters per la sottofamiglia Cracinae (Galliformes). Annali del Museo Civico di Storia Naturale Giacomo Doria, 79: 330-372.

Taibel A. M., 1974 - Esperimenti ibridologici nel genere Ortalis Merr. (Galliformes, Cracidae): ibridi fertili Ortalis guttata x O. canicollis. Rivista Italiana di Ornitologia, 44: 250-271.

Taibel A. M., 1974 - Recensione di Curassows and related birds di J. Delacour and D. Amadon. Rivista Italiana di Ornitologia, 44: 222-223.

Taibel A. M., 1975 - La "giogaia" del Tacchino di boscaglia (Alectura lathami J.E. Gray - Galliformes Megapodiidae) rappresenta un organo di recettività del senso termico e quindi utilizzato come regolatore della temperatura che si sviluppa nel nido-incubatrice? Rivista Italiana di Ornitologia, 45: 335- 341.

Taibel A. M., 1975 - Concordanze e divergenze nei due lavori di Vaurie e di Delacour \& Amadon sulla famiglia Cracidae (Galliformes). Discussioni e conclusioni personali. Atti della Società Italiana di Scienze Naturali e del Museo Civico di Storia Naturale in Milano, 116: 126-152.

Taibel A. M., 1976 - Caratteristiche e peculiarità anatomiche nei vari membri della famiglia Cracidae. Ricerche di Biologia della Selvaggina, 7: 677-726.

Taibel A. M., 1977 - Mammiferi del Guatemala, con speciale riguardo alla regione del Petèn, raccolti dal maggio al settembre 1932. Atti della Società Italiana di Scienze Naturali e del Museo Civico di Storia Naturale in Milano, 118: 379-403.

Taibel A. M., 1977 - Una nuova varietà di colore - ardesia-pallido - nel piumaggio del Cigno nero (Cygnus atratus ) sorta per mutazione fattoriale. Rivista Italiana di Ornitologia, 47: 282286.
Taibel A. M., 1979 - Leggende di uccelli. Buti, La grafica pisana, Pisa.

Taibel A. M., 1980 - Esperimento ibridologico fra specie di due generi distinti: Penelope (Merrem) e Ortalis (Merrem). Ibrido tetra specifico-digenerico [(Penelope pileata $\mathrm{x}$ P. argyrotis) $\mathrm{x}$ Penelope superciliaris] x Ortalis canicollis (Galliformes Cracidae). Rivista Italiana di Ornitologia, 50: 35-49.

\section{REFENCES}

Benazzi M., 1984 - Ricordo di Alulah Taibel (1892-1984). Bollettino di Zoologia, 51: 271-272.

Bollini G. \& Bragatto D., 2019 - Dal Podgora alle Ambe, dal Carso al Guatemala: la vita avventurosa di Alula Taibel, "ardito" e naturalista. [Autoprodotto].

Gippoliti S, \& Kitchener A.C., 2007 - The Italian zoological gardens and their role in mammal systematic studies, conservation biology and museum collections. Hystrix Italian Journal of Mammalogy, 18: 173-184.

Gippoliti S. \& Violani C., 2009 - Collezioni animali viventi e collezioni museali in Italia: un'opportunità persa? Museologia Scientifica Memorie, 4: 131-136.

Joseph L., Slikas B., Rankin-Baransky K., Bazartseren B., Alpers D., Gilbert A.E., 1999 - DNA evidence concerning the identities of Crax viridirostris Sclater, 1875, and C. estudilloi Allen, 1977. Ornitologia Neotropical, 10: 129-144.

Kimball R. T., Braun E. L. \& Ligon J. D., 1997 - Resolution of the phylogenetic position of the Congo peafowl, Afropavo congensis: a biogeographic and evolutionary enigma. Proceedings of the Royal Society London B, 264: 1517-1523.

Ralls K. \& Ballou J., 1983 - Extinction: Lessons from zoos. In: Genetics and Conservation: a reference for managing wild animal and plant populations. C.M. Schonewald-Cox, S.M. Chambers, B. MacBryde \& L. Thomas (eds.). Benjamin/Cummings, Menlo Park, CA.: 164-184.

Taibel A. M., 1937 - L'antilope cervicapra: osservazioni sul gruppo in allevamento presso la Stazione sperimentale di Avicoltura di Rovigo. Rassegna Faunistica, 4: 3-19.

Taibel A. M., 1950 - Osservazioni su alcuni neonati di capibara (Hydrochoerus hydrochoeris). Cesalpinia, gennaio: 29-47.

Taibel A. M., 1954 - Sistematica e Ibridologia. Generi, specie, sottospecie, razze biologiche nella Sottofamiglia Cracinae. Bollettino di Zoologia, 21 (2): 261-272.

Taibel A. M., 1962 - L'Acquario-Rettilario dello Zoo di Torino. Natura e Montagna, 2: 133-138.

Taibel A. M., 1971 - "Tibie piegate ad angolo", mutazione condizionata da un fattore "letale" in Crax e in Mitu (Galliformes - Cracidae). Natura, 62 (2): 197-209.

Taibel A. M., 1977 - Mammiferi del Guatemala, con speciale riguardo alla regione del Petèn, raccolti dal maggio al settembre 1932. Atti Società italiana di Scienze Naturali, 118 (3-4): 379-403. 